\title{
Visualizing Phase Transition Induced Actuation in Vanadium Dioxide in a Transmission Electron Microscope
}

\author{
B.Viswanath and Shriram Ramanathan
}

Harvard School of Engineering and Applied Sciences, Harvard University Cambridge, MA, 02138

We demonstrate a novel approach to visualize, image and quantify actuation in nanostructures that undergo structural transitions by Fresnel contrast imaging of electron transparent cantilevers. Quantitative demonstration of the approach to measure the cantilever deflection in situ is presented for a model correlated insulator vanadium dioxide that undergoes a sharp monoclinic-tetragonal transition near $68^{\circ} \mathrm{C}$, and a material of interest in oxide electronic devices, electro mechanical actuators and strain sensors. Quantification of the stress relaxation from in-situ TEM studies is then directly compared to macroscopic stress relaxation from wafer curvature measurements showing how one can investigate lattice relaxation across length scales. The measurement allows direct comparison between the structural phase transition and actuation in a nanostructured material form.

Direct visualization of the temperature driven, phase transition induced actuation in $\mathrm{VO}_{2}$ cantilevers enable the quantification of cantilever deflection with sub $70 \mathrm{~nm}$ resolution. Abrupt, reversible cantilever tip deflection of $550 \mathrm{~nm}$ consistent with the estimated $250 \mathrm{MPa}$ stress (tensile) development is observed across the monoclinictetragonal structural transition temperature of $68^{\circ} \mathrm{C}$. Smooth negative deflection changes are seen with different slopes at below and above transition temperature corresponding to thermal mismatch stress of insulating and metallic phases of $\mathrm{VO}_{2}$. This method provides the possibility of probing the structural/microstructural changes and the corresponding actuation in terms of cantilever tip deflection from the same regions often unachievable through other ex situ means especially from the nanoscale cantilevers. The high resolution capabilities of TEM is well suited for measuring the deflection from electron transparent nanocantilevers and enable the deflection measurement from $\mathrm{VO}_{2}$ cantilevers of varied dimensions from 5 micron to $50 \mathrm{~nm}$ in length.

The electron optics ray diagram along with the experimental protocol to measure the cantilever deflection is shown in Figure 1. The set up is same of any conventional TEM with heating capabilities with the only difference being the use of temperature induced dynamic cantilever in the place of static TEM specimen. The maximum cantilever tip deflection can be directly observed at the cantilever edge with Fresnel contrast by tracing the defocus or specimen height. The measured cantilever deflection during the thermally induced phase transition is shown in Figure 2. The presence of Fresnel fringes around the edge of cantilever enables visualization of cantilever motion at few tens of nanometer resolution (Figure 3). The upward motion of the cantilever across the monoclinic-tetragonal transition is evident from the abrupt changes in Fresnel contrast. This method is well suitable for smaller cantilevers and more importantly, enable the observation of structural/microstructural changes from the same region aiding the direct mechanistic understanding of actuation. 


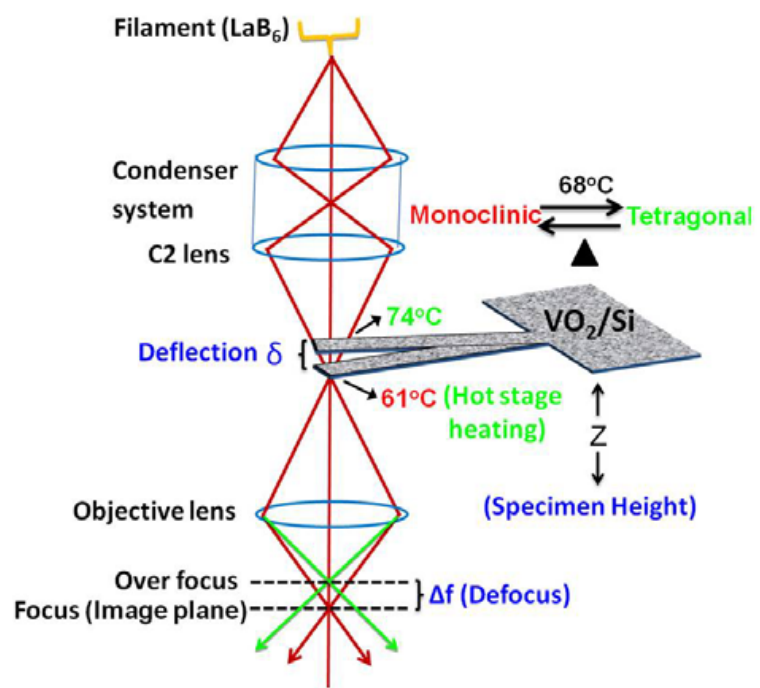

Fig 1. Schematic to visualize and measure the phase transition induced actuation in $\mathrm{VO}_{2}$ cantilever.

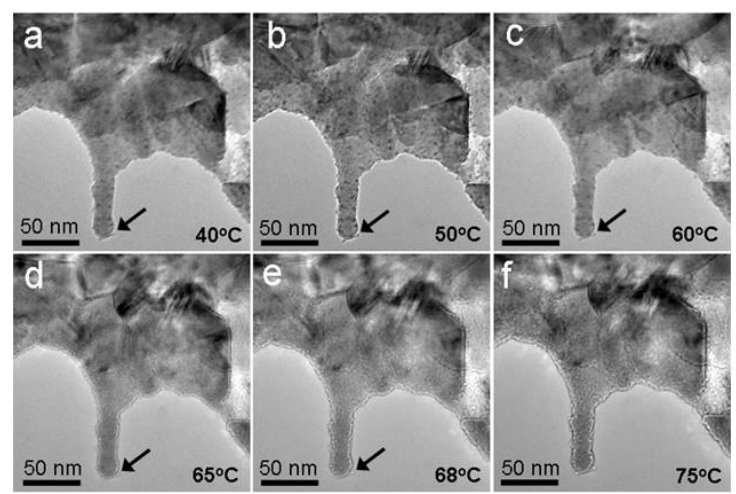

Fig 3. Visualization of phase change induced actuation via in situ Fresnel contrast imaging.
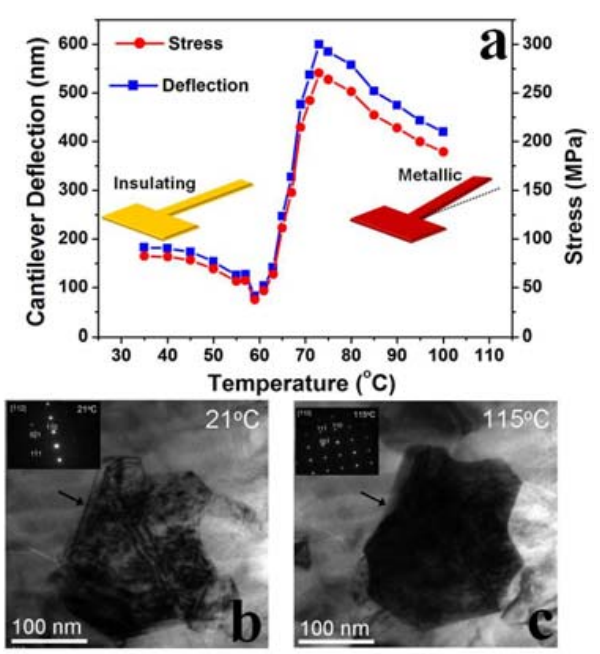

Fig 2. $\mathrm{VO}_{2}$ cantilever deflection measurement across the monoclinic-tetragonal phase transition. 